Supporting Information to

\title{
The Role of Delocalization and Excess Energy on the Quantum Efficiency of Organic Solar Cells and the Validity of Optical Reciprocity Relations
}

N. Felekidis ${ }^{1}$, A. Melianas ${ }^{2}$ and M. Kemerink ${ }^{1,3}$

${ }^{1}$ Complex Materials and Devices, Department of Physics, Chemistry and Biology, Linköping University, 58183 Linköping, Sweden

${ }^{2}$ Department of Materials Science and Engineering, Stanford University, Stanford, California 94305, USA

${ }^{3}$ Centre for Advanced Materials, University of Heidelberg, 69120 Heidelberg, Germany.

Email: martijn.kemerink@cam.uni-heidelberg.de

\section{Contents}

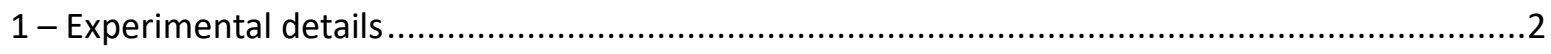

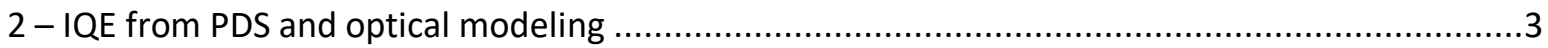

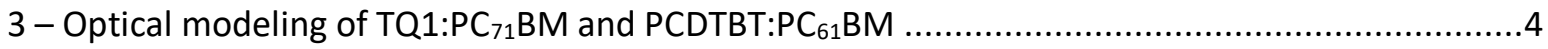

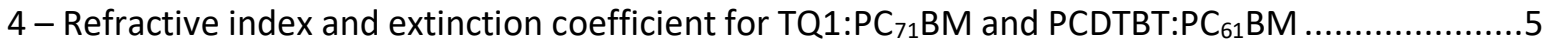

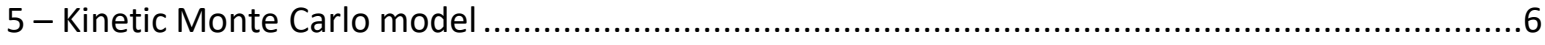

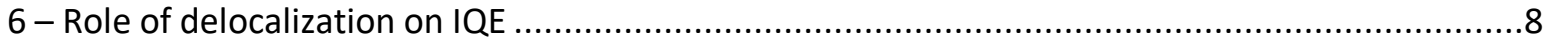

$7-$ CT-EL and absorption fits according to Marcus model.............................................................

8 - JVs under 1 Sun AM1.5 illumination for TQ1:PC71BM and PCDTBT:PC61BM .........................10

9 - Reciprocity analysis of experiment and model fits .............................................................11

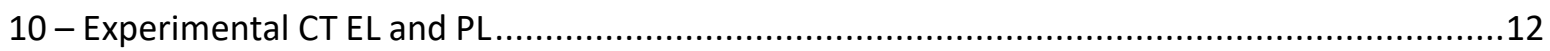

11 - Parameters for kMC simulations and absorption calculations in the main text.....................13

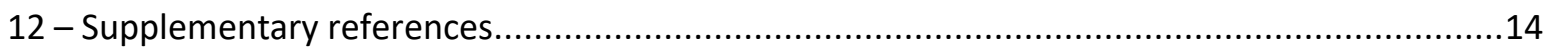




\section{1 - Experimental details}

Device fabrication Bulk heterojunction OPVs where made from polymer-fullerene solutions of $25 \mathrm{~g} / \mathrm{L}$ concentration in 1,2-dichlorobenzene (ODCB)according to the following process. A $40 \mathrm{~nm}$ thin film of poly-(3,4-ethylenedioxythiophene) polystyrene sulfonate (PEDOT:PSS) was spin coated on pre-cleaned ITO/glass substrates in air. After baking, the active layer was spin coated in a glove box. A 0.6/90 nm $\mathrm{LiF} / \mathrm{Al}$ top contact was evaporated on the active layer under high vacuum conditions. The device areas for all devices were measured to be $0.044-0.048 \mathrm{~cm}^{2}$ while the thicknesses $80 \mathrm{~nm}$ and $90 \mathrm{~nm}$, for TQ1:PC71BM and PCDTBT:PC61BM respectively. Samples were encapsulated in the glovebox directly after fabrication using glass cover slides and UV-cured glue.

Fourier Transform Photocurrent Spectroscopy FTPS-EQE measurements EQE vs applied voltage was measured from FTPS using an IR Vertex 70 vacuum spectrometer for both TQ1:PC ${ }_{71} B M$ and $\mathrm{PCDTBT}: \mathrm{PC}_{61} \mathrm{BM}$. The resulting spectra were calibrated using a reference Si Photodiode (Hamamatsu S1133-01). The resulting EQE-FTPS spectrum was scaled to a reference EQE, measured with an Enlitech QE-R Solar Cell Spectral Response Measurement System for TQ1:PC ${ }_{71} B M$ and PCDTBT:PC 61 BM. The resulting EQE was scaled to a reference Si Photodiode. The same equipment was used to measure reflection using a STAN-SSH high-reflectivity specular reflectance standard.

Variable Angle Spectroscopic Ellipsometry (VASE) Spectroscopic ellipsometry measurements were performed in the spectral range $245-1690 \mathrm{~nm}$ at angles of incidence $45,55,65$ and $75^{\circ}$ with an RC2 ellipsometer from J.A. Woollam Co., Inc. (USA). The software (CompleteEASE, J.A. Woollam Co., Inc.) was used to model the optical constants of TQ1:PC ${ }_{71} B M$ and PCDTBT:PC ${ }_{61} B M$ thin films spin coated on silicon substrates, using Kramers-Kronig consistent B-spline models. ${ }^{1}$ The thin films were processed correspondingly to the device active layers. The PCDTBT:PC ${ }_{61} \mathrm{BM}$ film was strongly scattering and impossible to fit adequately; hence the ellipsometry measurement was complemented with transmittance and reflectance measurements.

UV/Vis UV/Vis transmittance and reflectance of TQ1:PC $\mathrm{C}_{71} \mathrm{BM}$ and $\mathrm{PCDTBT}: \mathrm{PC} \mathrm{C}_{61} \mathrm{BM}$ was measured with a Perkin Elmer Lambda 900 UV-Vis-NIR spectrophotometer. From the Beer-Lambert law and film thickness measured by an Dektak profilometer (Veeco Stylus 6M) the extinction coefficient of the PCDTBT:PC 61 BM was calculated.

Optical simulations A home written TMM Matlab script was used for optical simulations. For TQ1:PC ${ }_{71} \mathrm{BM}$ optical constants were determined by VASE and for PCDTBT:PC ${ }_{61} B M$ the extinction coefficient was determined from UV/Vis by the Beer-Lambert law and the refractive index was fixed to $2 .^{2}$

Photothermal Deflection Spectroscopy (PDS) PDS measurements were performed on a home-built setup described by Vandewal et al. ${ }^{3,4}$ Measurements were taken using a mechanically chopped $(3 \mathrm{~Hz})$ monochromatic light source using both a 150W Xenon and 100W halogen pump lamp with $5 \mathrm{~nm}$ spectral resolution focused onto the sample. Degassed and filtered perflourohexane (C6F14, 3M Flourinert FC-72) was used as the deflection medium. Thin films were made in a glovebox prepared vida supra by spincoating on quartz substrates without metal contacts in a nitrogen environment with $<1 \mathrm{ppm}$ of oxygen. The sample was aligned with maximal overlap between the pump beam and thin film. Samples were normalized for extinction above gap via procedure described in Ref. ${ }^{5}$.

CT Electroluminescence and Photoluminescence CT Electroluminescence and Photoluminescence were measured with a Shamrock 303i spectrograph (Andor Tech) using a Newton EM-CCD Si array as the detector. Photo-excitation was performed with a 400nm laser during CT-PL while an external filed was applied to the device via a Keithley 2400 source-measure unit. 
2 - IQE from PDS and optical modeling
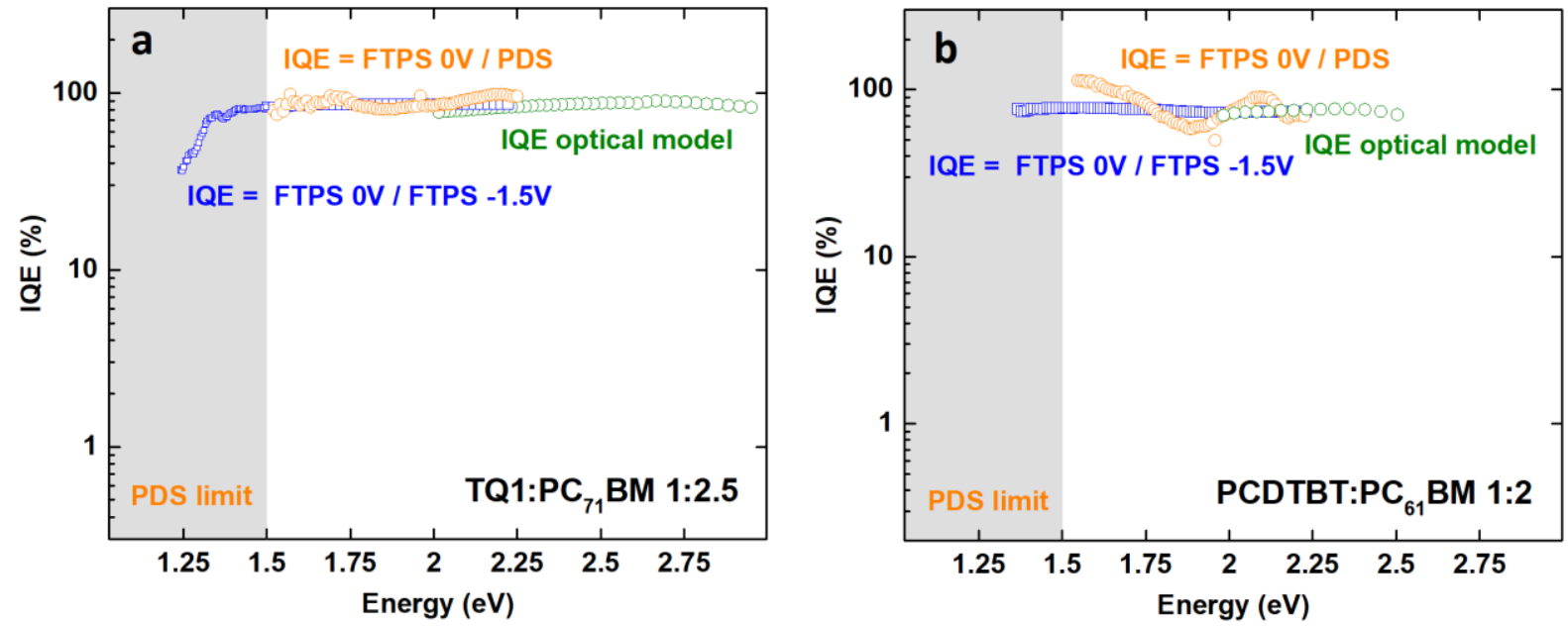

Figure S1. Experimental IQE (OV) calculated as FTPS (OV) / FTPS (-1.5V) (blue trace) and as FTPS(OV)/PDS for a) TQ1:PC ${ }_{71} B M$ and $\left.b\right)$ PCDTBT:PC 61 BM. Green traces are TMM simulated IQE spectra with optical modeling of the full device stack and were used as a scaling reference of the absolute IQE value. 

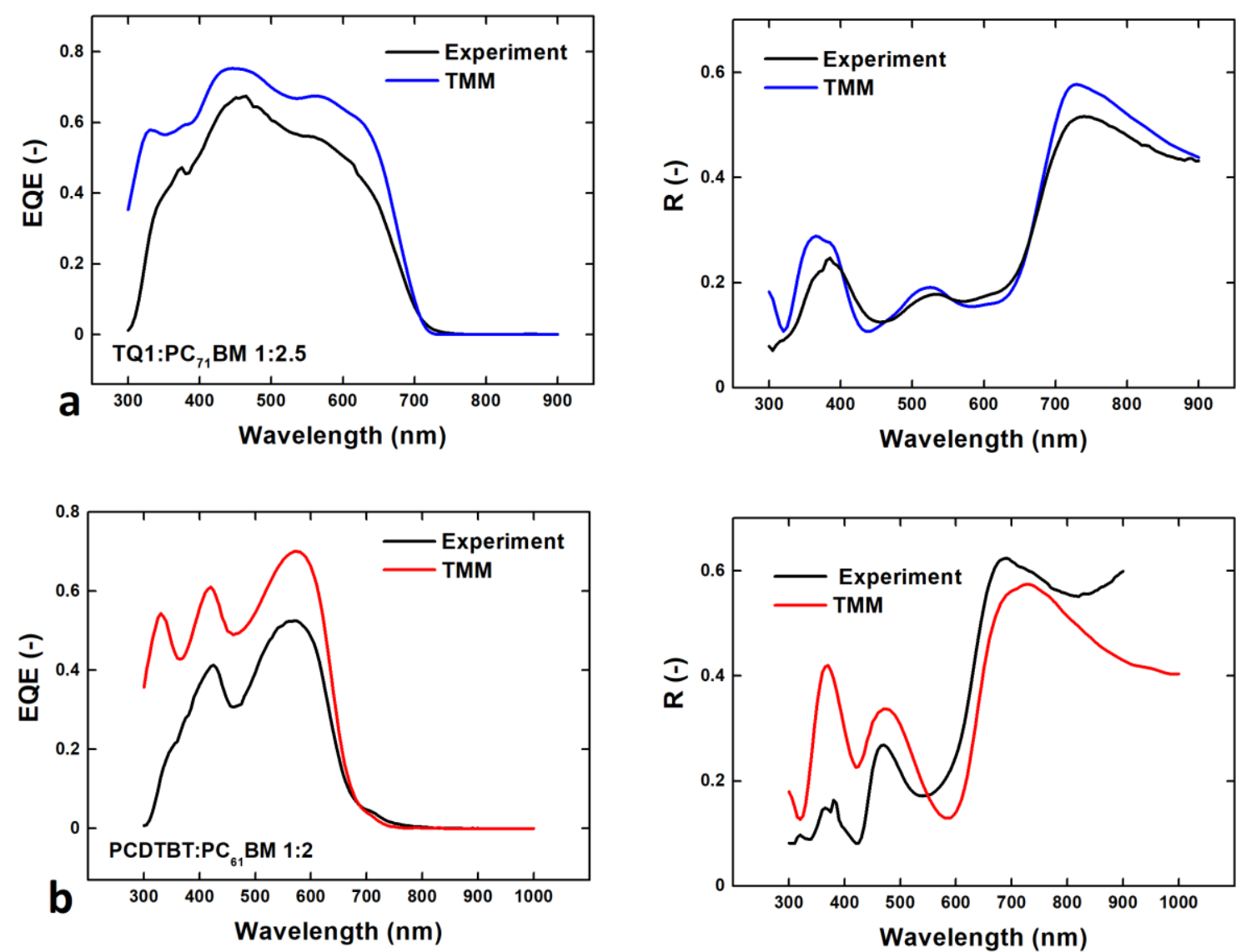

Figure S2. Experimental and transfer matrix modeled (TMM) EQE (left) and full device reflectance (right) for a) TQ1:PC ${ }_{71} B M$ and b) PCDTBT:PC ${ }_{61} B M$ for $75 \mathrm{~nm}$ thick active layers. Reflectance was measured using an integrating sphere. The limited quality of the reflectance fit for the PCPDTBT:PC ${ }_{61} B M$ sample is due to the sample being thin and scattering. 
4 - Refractive index and extinction coefficient for TQ1:PC ${ }_{71} \mathrm{BM}$ and PCDTBT:PC ${ }_{61} \mathrm{BM}$
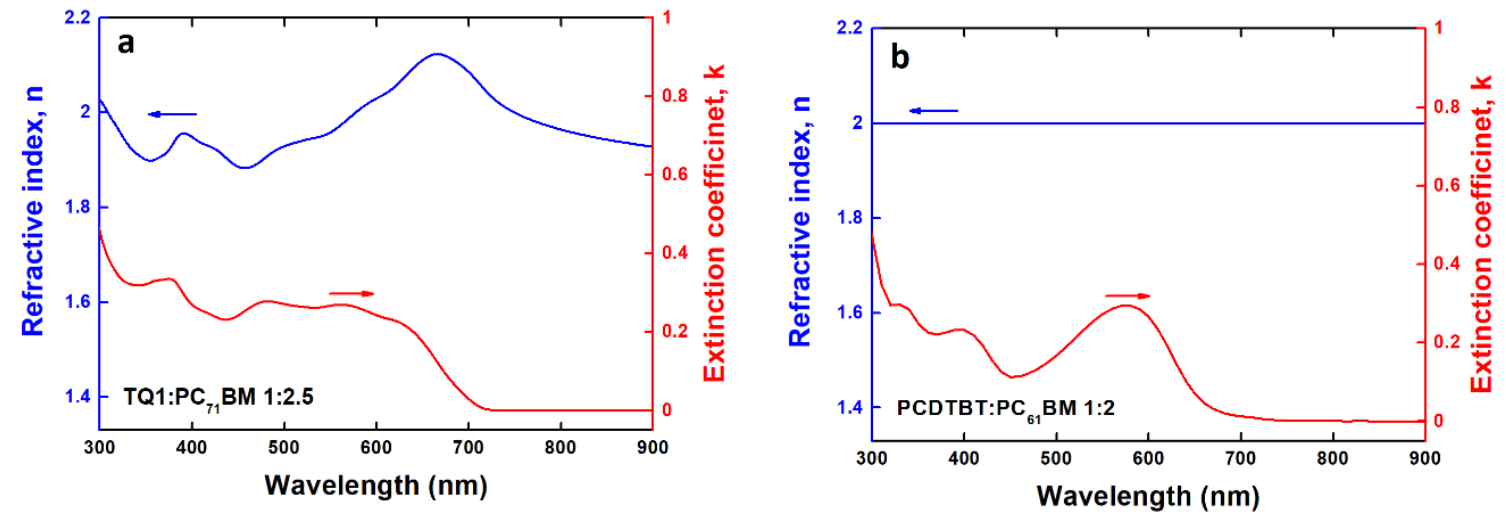

Figure S3. Experimental refractive index $n$ and extinction coefficient $k$ for a) TQ1:PC ${ }_{71} B M$ and b) PCDTBT:PC ${ }_{61} B M$. The refractive index for PCDTBT could not be measured with ellipsometry as the sample was thin and scattering. 


\section{5 - Kinetic Monte Carlo model}

The kinetic Monte Carlo model describes charge transport of electrons and holes as a thermally activated tunneling (hopping) process on a 3D cubic lattice with inter-site distance $a_{N N}$, where the rates for each hop are calculated by the Miller-Abrahams expression. ${ }^{6}$ The model accounts for Coulomb interactions between all the charges in the device and incorporates intrinsic material parameters such as static energetic disorder, energy levels of the effective medium (donor HOMO and acceptor LUMO), hopping rates, $\mathrm{CT} /$ exciton recombination rates and the dielectric constant. ${ }^{6}$ The simulation focuses on the 'long-range' charge separation acting on $>$ ps timescales. ${ }^{7}$ Hence, it is assumed that all ultrafast (faster than $1 \mathrm{ps}$ ) processes have been completed at the start of the simulation. These include in particular exciton dissociation by (partially) coherent electron/hole motion (delocalization) and on-site thermalization as well as polaron formation, which have been shown to occur predominantly at sub-ps time scales. ${ }^{7,8}$ Hence, as shown in Figure 2 , at the beginning of the simulation an on-site thermalized electron-hole pair is created, which can undergo inter-site thermalization, while the effects of delocalization are in lowest order approximated by an enhanced initial separation. Since we assume that ultrafast processes including delocalization have completed prior to the simulation start, an initially 'delocalized' electron-hole pair that reforms an exciton or nearest-neighbor $\mathrm{CT}$ exciton does not again delocalize, but it can still re-dissociate via electron or hole hopping within its lifetime. Likewise, CT pairs formed in EL simulations cannot undergo the same initial (ultrafast) carrier separation as photogenerated carriers.

Regarding the excitation energy, the electron (e) and hole (h) are generated at random energies in the LUMO and HOMO levels under the condition that $E_{e}-E_{h}=E_{\text {photon, }}$ assuming equal absorption probability for all electron and hole sites. The weighing function of this excitation transition is the joint DOS given by:

$$
g_{e-h}\left(E_{\text {photon }}\right)=g_{h}\left(E_{h}\right) * g_{e}\left(E_{h}-E_{\text {photon }}\right)
$$

where $*$ denotes convolution. Here, we tacitly assume that any energy loss of the exciton prior to charge transfer can be ignored for the following reason. Although unimportant once a CT state has been formed, i.e. prior to the starting point of the present simulations, Förster and Dexter processes are included in the simulation model. Dexter is implicitly included as a two-step charge motion process (electron hop followed by hole-hop or vice versa); Förster is explicitly included with a Förster radius of $\sim 4 \mathrm{~nm}$, following our previous work. ${ }^{9}$ In that work, we found that in the well-mixed blends considered herein, Förster transfer is not important due to the much higher rate for downward charge hopping than for exciton recombination. Likewise, in well-mixed blends only few, if any, Dexter steps are needed to reach an interface. For the same reason as that charge carrier relaxation in the DOS requires many steps to complete, c.f. Ref. ${ }^{9}$, these few 'exciton hops' do not cause a significant energy loss; significant in this context means reaching energies at which charge transfer and subsequent long-range separation would be negatively affected - that is, energies that are deep in the joint DOS. The same argument applies to Förster transfer.

At the beginning of each simulation, a single electron-hole CT pair with random $E_{e l}, E_{h o}$, linked by condition (2), is created. The simulation terminates when any of the two charge carriers reaches a contact or if the carriers recombine. Since non-geminate recombination is negligible at the considered short circuit conditions (IQE $~ 90 \%$ ), ${ }^{10}$ recombination with other charges can be ignored. Averaging multiple independent simulations for different excitation energies and different initial separation distances, denoted as CT $n$ where $n-1$ is the number of sites between the electron and hole at the start of the simulation (e.g. CT1 is the nearest-neighbor pair), results in the simulated IQE spectrum. As lowest, and for the current purposes sufficient approximation we take the initial separation to be 
independent of excitation energy, as suggested by the calculations in Ref. ${ }^{7}$. Only electron hole pairs that are situated on the same site (an exciton) or situated on nearest neighbor sites (CT1) can recombine. The initial orientation of the CT pair with respect to the direction of the electric field was found to have a negligible effect on the resulting IQE spectra and is ignored; see Figure S4 for more details. For simplicity, a generic but experimentally representative set of disorder parameters is used for both systems and only the energy levels and the hopping and CT-recombination rates are adjusted for each system. The full set of used parameters and literature sources for them is given in Table S1.
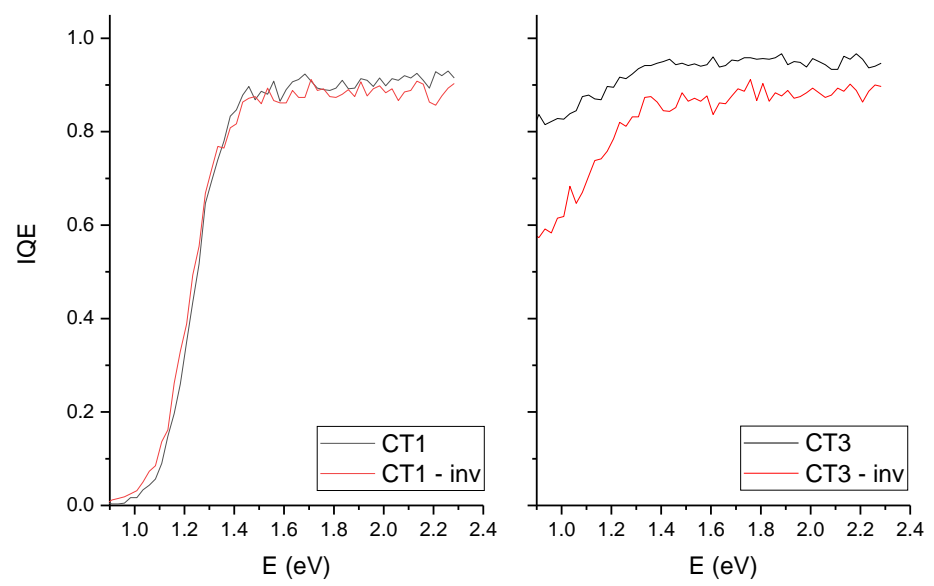

Figure S4. Effect of initial electron-hole orientation on the IQE. Kinetic Monte Carlo simulations of IQE vs. excitation energy are performed for different initial e-h orientations along the z-axis, i.e. the electron-hole pair is either placed parallel (black) or antiparallel (red) to the field for a) one site (CT1) and b) three sites (CT3) initial delocalization lengths. Material parameters are taken as for TQ1:PCBM, see Table S1. 

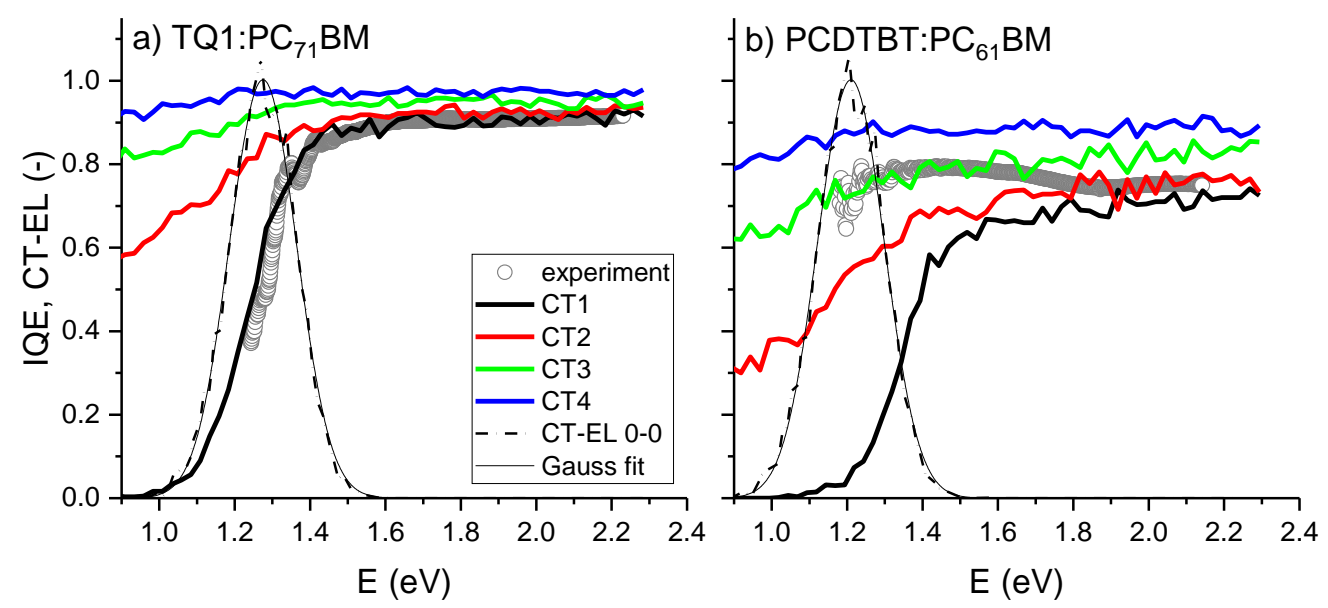

Figure S5. IQE vs excitation energy spectrum from kinetic Monte Carlo simulations (heavy solid lines) parametric in initial e-h separation distance for (a) TQ1:PCBM and b) PCDTBT:PCBM. Dash-dotted thin lines are raw simulated 0-0 CT-EL spectra, thin solid lines are Gaussian fits to those. CTn denotes initial separation distance, with $n-1$ the number of sites between the electron and hole (e.g. CT1 is the nearest-neighbor pair).

The role of initial delocalization on the excitation energy dependence of the IQE was further studied using kinetic Monte Carlo simulations. In the simulations, the initial CT charge pair separation was varied from $\mathrm{CT} 1$ to $\mathrm{CT} 4$. The results are shown in Figure $\mathrm{S} 5$, together with the corresponding simulated $C T-E L_{0-0}$ spectra. Independent of initial separation, and in agreement with the experimental findings, the model predicts efficient and photon energy-independent charge separation at excitation energies that are significantly above the CT-EL $\mathrm{L}_{0-0}$ emission peak, c.f. the red arrow in Figure 2.

At lower excitation energies, where the electron and the hole are deep in the tail of the joint DOS, as shown by the blue arrow in Figure 2, the efficiency of charge separation is strongly determined by the initial delocalization of the CT pair. Although excitation at the CT-EL $\mathrm{L}_{0-0}$ emission peak is, for all initial separations, rather deep in the joint DOS, there is still ample room for further relaxation which helps to overcome the Coulomb binding energy. At even lower excitation energies, relaxation is no longer sufficient to overcome the attractive Coulomb barrier and the IQE drops towards zero. The roll-off energy is dominated by the Coulomb barrier th\{Citation\}at is to be overcome, set by the initial separation, which is in part governed by charge delocalization. The balance between the CT recombination rate on the one hand and the hopping rates on the other has a smaller influence on the roll-off energy. These results therefore suggest that the energy-dependence of IQE at low photon energies could be used as an indicator for the extent of charge delocalization.

Finally, the question naturally arises how delocalization, for which the initial separation CTn is used as a proxy, relates to material parameters and especially morphology. Following previous literature in which it was argued that the presence of fullerene domains promotes charge separation, we hypothesize that that the observed difference between the TQ1 system (CT1) and PCDTBT system (CT3) reflects a difference in PCBM aggregation. ${ }^{11-13}$ Indeed, for the PCDTBT:PCBM system there are direct and indirect indications of PCBM aggregation that could facilitate ultrafast charge delocalization. ${ }^{12-15}$ For the TQ1 system, the available literature suggests that PCBM aggregation in unannealed films, as used here, is suppressed, in line with the current findings. ${ }^{16}$ 
The following fits where done according to equations 1,2 in Ref. ${ }^{17}$ :

$$
\begin{gathered}
\sigma(E) E=\frac{f_{\text {absorption }}}{\sqrt{4 \pi \lambda k T}} \exp \left(\frac{-\left(E_{C T}+\lambda-E\right)^{2}}{4 \lambda k T}\right) \\
\frac{I_{f}}{E}=\frac{f_{\text {emission }}}{\sqrt{4 \pi \lambda k T}} \exp \left(\frac{-\left(E_{C T}-\lambda-E\right)^{2}}{4 \lambda k T}\right)
\end{gathered}
$$
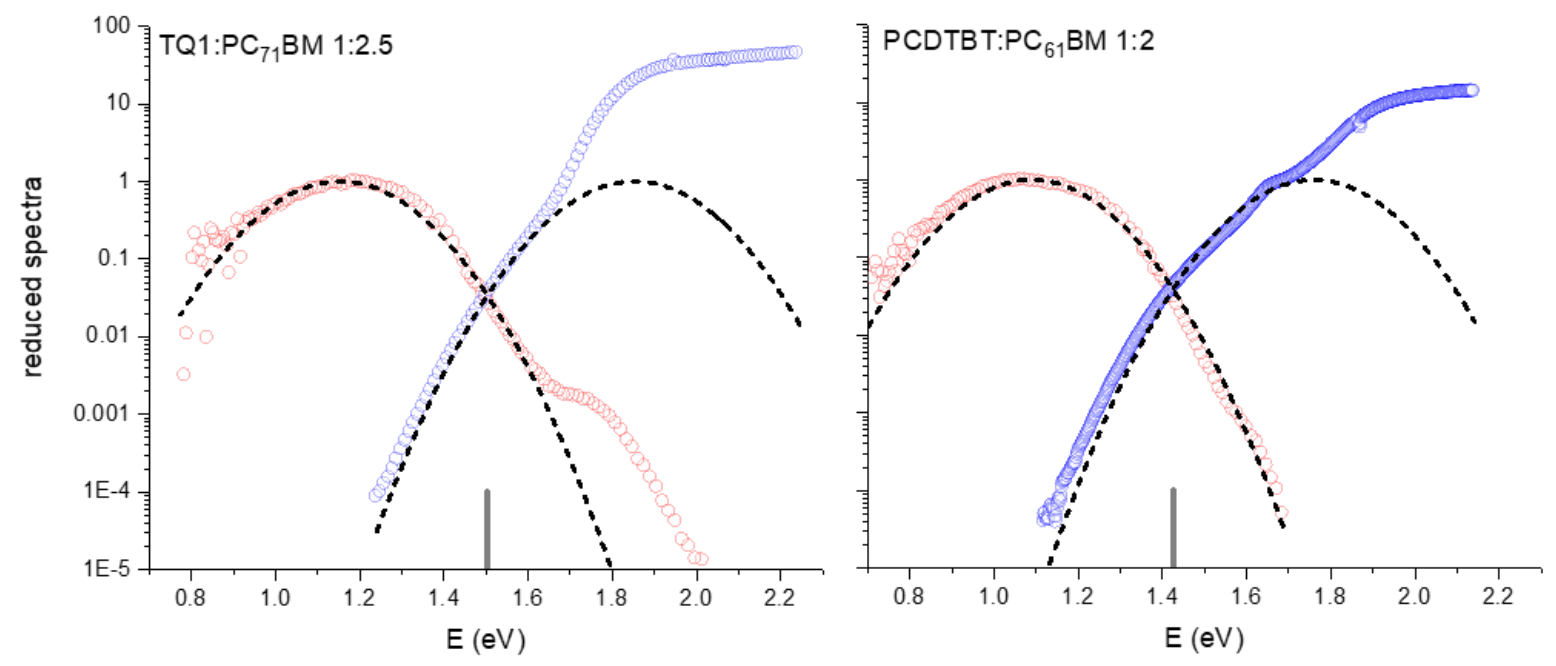

Figure S6. CT electroluminescence (red symbols) and EQE (blue symbols) fitted with equations 1, 2 for a) TQ1:PC ${ }_{71} B M$ and $b$ ) PCDTBT:PC 61 BM. The full spectrum was fitted using the same $\lambda$ for emission and absorption. The fitted parameters are $\lambda=0.35 \mathrm{eV}$ and $0.335 \mathrm{eV}$ and $E_{C T}=1.503 \mathrm{eV}$ and $1.425 \mathrm{eV}$ for TQ1:PC ${ }_{71} \mathrm{BM}$ and PCDTBT:PC 61 BM, respectively. 
8 - JVs under 1 Sun AM1.5 illumination for TQ1:PC71BM and PCDTBT:PC61BM

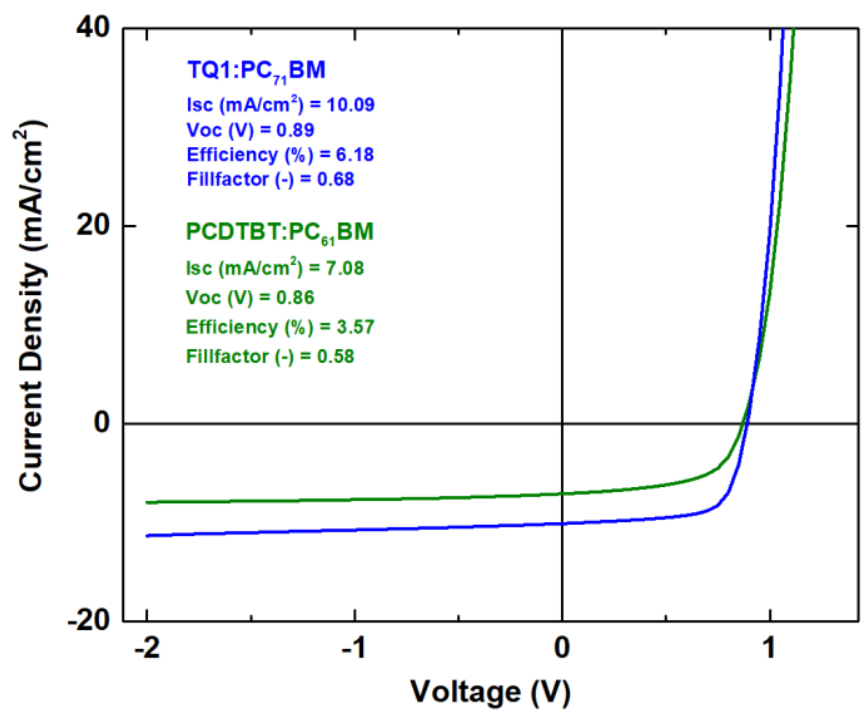

Figure S7. Experimental current density vs applied voltage under constant 1Sun AM.1.5 illumination for TQ1:PC ${ }_{71} \mathrm{BM}$ and PCDTBT:PC ${ }_{61} \mathrm{BM}$. 

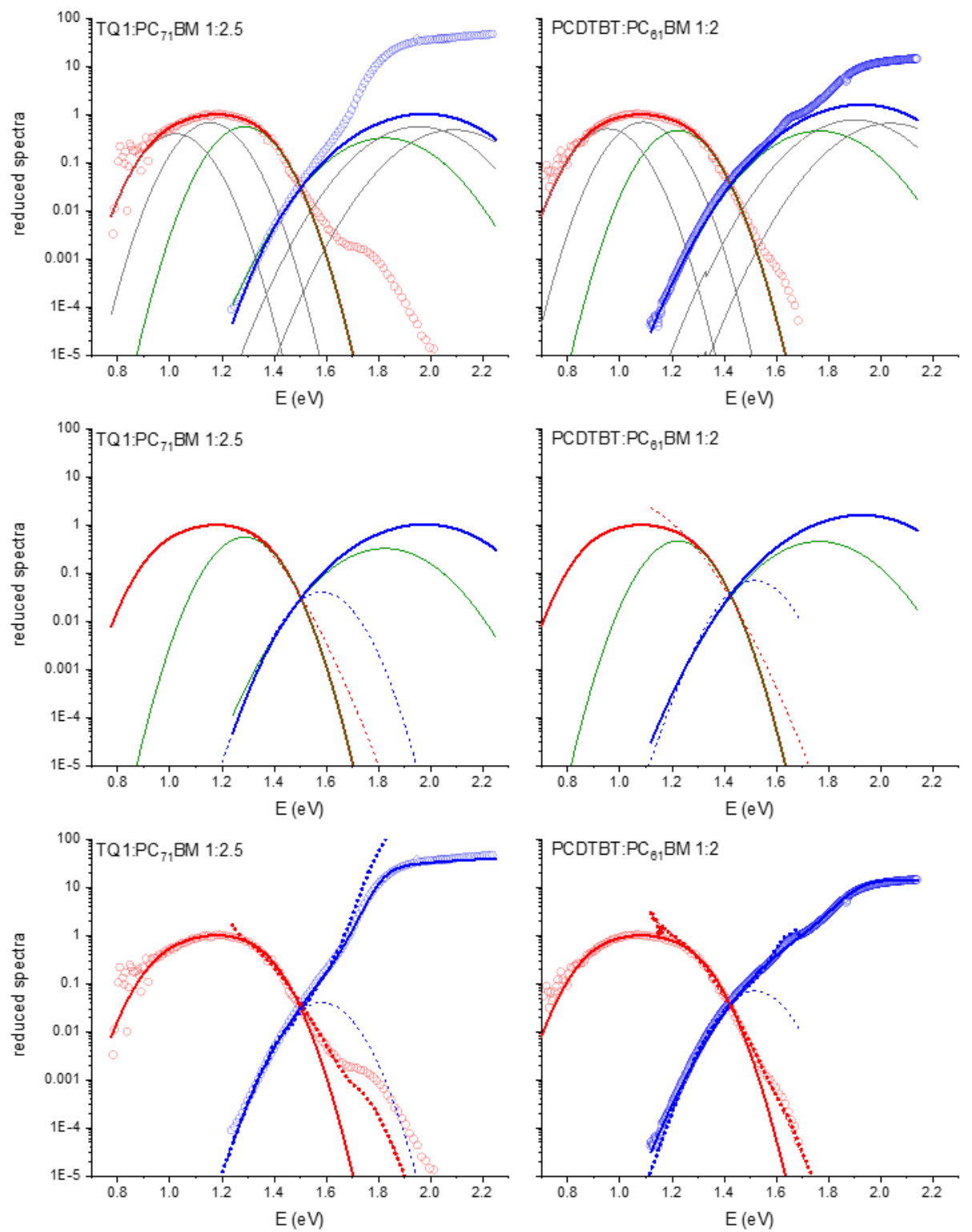

Figure S8. Reciprocity analysis of the experimental and simulation data in Fig. 3 of the main text for TQ1:PCBM (left) and PCDTBT:PCBM (right). The top panels show the experimentally measured CT-EL (red symbols) and EQE (blue symbols) spectra. KMC simulations are shown as lines: thin green - 0-0 CT-EL and 0-0 absorption spectra; solid red and blue lines are the sums of the vibronic manifolds thereof for CT-EL and CT-EQE, respectively. EQE spectra are obtained by multiplying the corresponding absorption spectrum with the calculated IQE spectrum. The middle panels show the reciprocity analysis for the simulation curves in the top panel. Dashed curves are obtained by dividing (multiplying) the CT-EL (EQE) spectra by the black body spectrum at $300 \mathrm{~K}$. Note that the width of the spectra obtained in this manner does not match that of the corresponding simulated spectra, cf. dashed and solid lines of the same color. The lower panels show the reciprocity analysis for the experimental data and the total simulated EQE spectra with heavy and thin dotted lines obtained respectively from experimental and simulated curves via the $300 \mathrm{~K}$ black body spectrum; here, the solid blue line is the full $\mathrm{kMC}$ EQE spectrum, including a phenomenological Urbach tail. Clear deviations are present away from the intersection region. Note that typically the high-energy data where S1 emission and absorption sit are not shown/considered. 
10 - Experimental CT EL and PL
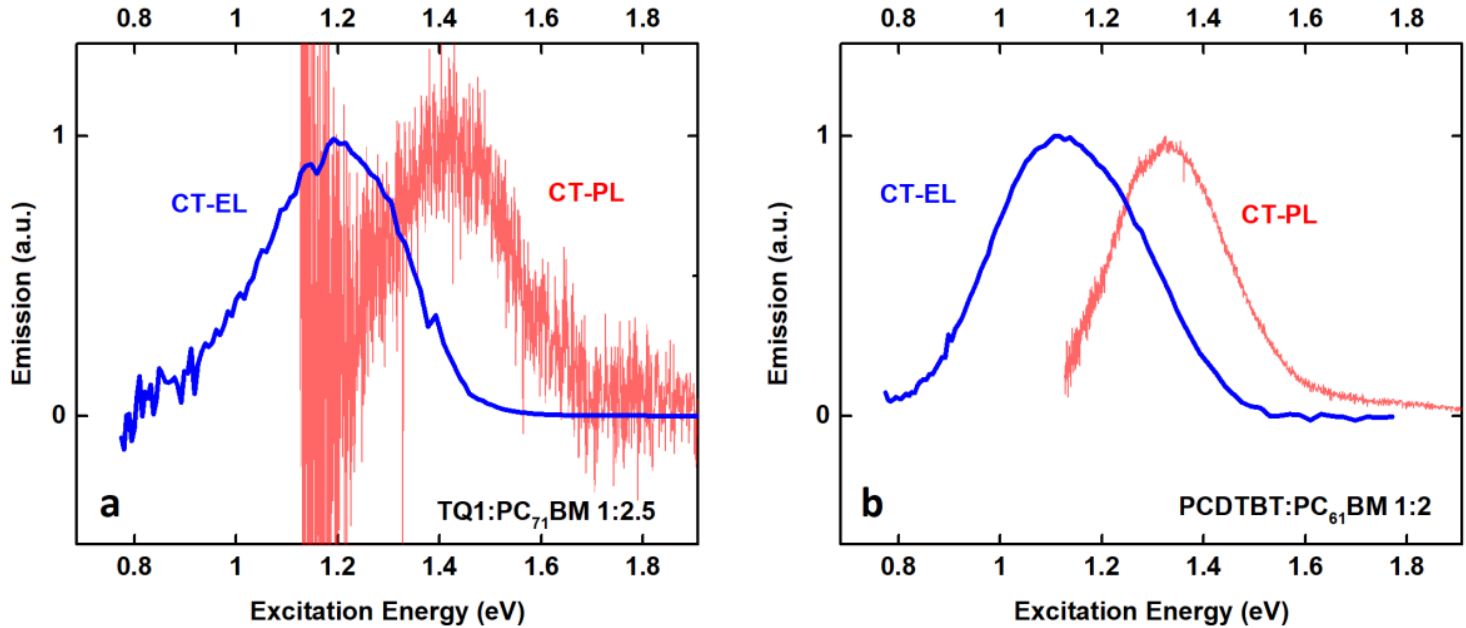

Figure 59. Experimental CT electroluminescence (blue traces) and CT photoluminescence (red traces) for a) TQ1:PC ${ }_{71} \mathrm{BM}$ and b) PCDTBT:PC ${ }_{61} \mathrm{BM}$. CT-EL was measured at $1.15 \mathrm{~V}$ (current density $25 \mathrm{~mA} / \mathrm{cm}^{2}$ ) for TQ1:PC $\mathrm{C}_{71} \mathrm{BM}$ and $2.64 \mathrm{~V}\left(200 \mathrm{~mA} / \mathrm{cm}^{2}\right)$ for PCDTBT:PC 61 BM. The CT-PL spectra were obtained by subtracting the PL spectra of the OPV device at short-circuit conditions from the PL spectra at open-circuit conditions. 


\section{1 - Parameters for kMC simulations and absorption calculations in the main text}

\begin{tabular}{|c|c|c|}
\hline & TQ1:PC ${ }_{71} \mathrm{BM}$ & PCDTBT:PC ${ }_{61} B M$ \\
\hline$E^{L U M O}[\mathrm{eV}]$ & \multicolumn{2}{|l|}{-3.9} \\
\hline$E^{\mathrm{HOMO} O}[\mathrm{eV}]$ & -5.7 & -5.6 \\
\hline$\sigma^{L U M O}[\mathrm{meV}]$ & \multicolumn{2}{|l|}{120} \\
\hline$\sigma^{\mathrm{HOMO}}[\mathrm{meV}]$ & \multicolumn{2}{|l|}{85} \\
\hline$v_{0}^{L U M O}\left[\mathrm{~s}^{-1}\right]$ & $1 \times 10^{13}$ & $3 \times 10^{12}$ \\
\hline$v_{0}^{\mathrm{HOMO}}\left[\mathrm{s}^{-1}\right]$ & $5 \times 10^{9}$ & $1 \times 10^{9}$ \\
\hline$v_{C T}\left[\mathrm{~s}^{-1}\right]$ & $8 \times 10^{7}$ & $2 \times 10^{8}$ \\
\hline$v_{e x}\left[\mathrm{~s}^{-1}\right]$ & \multicolumn{2}{|l|}{$1 \times 10^{9}$} \\
\hline$\alpha_{N N}[\mathrm{~nm}]$ & \multicolumn{2}{|l|}{1.8} \\
\hline built-in field [V/m] & $1.4 \times 10^{7}$ & $1.0 \times 10^{7}$ \\
\hline$\Delta \mathrm{E}_{\text {LUMO }}[\mathrm{eV}]$ & \multicolumn{2}{|l|}{0.6} \\
\hline
\end{tabular}

Table S1. Material parameters used for the kinetic Monte Carlo simulations shown in the main text. The CT-EL spectra are calculated for the (generic) TQ1:PCBM parameters with $V=V_{b i}=1 \mathrm{~V}$ and a thickness of $80 \mathrm{~nm}$. TQ1 parameters are taken from Ref. ${ }^{18}$, PCDTBT parameters are taken from Refs. ${ }^{9,19}$. The generic lattice constant $a_{N N}$ is additionally based on Ref. ${ }^{20}$. The generic LUMO level offset $\Delta \mathrm{E}_{\text {LUMO }}$ has no influence on the results as long as it exceeds the difference in exciton and CT binding energy (of about $0.3 \mathrm{eV}$ for the used parameters), which is the case as both systems have a large driving force.

It should be kept in mind that the slowness of kMC precludes any real least-squares fitting of (ideally several) experiments. This, in combination with the fact that there often exists an interchangeability between parameters (e.g. between disorder and hopping rate), makes that the presented parameter values should be considered approximate. Again, the slowness of kMC precludes estimation of a real error bar, but from experience we estimate the uncertainty in disorder values to be around 10-20 meV and in hopping rates about an order of magnitude or less. Importantly, precise numerical values have no impact on the conceptual conclusions of this work provided $\sigma \gg k_{B} T$, which is always the case.

\begin{tabular}{|l|l|l|}
\cline { 2 - 3 } \multicolumn{1}{c|}{} & TQ1:PC ${ }_{71} \mathbf{B M}$ & PCDTBT:PC $_{61} \mathbf{B M}$ \\
\hline$S$ & 1.5 & 1.9 \\
\hline$h \omega_{0}[\mathrm{meV}]$ & 133.7 & 136.3 \\
\hline CT-EL 0-0 peak width [meV] & 89.2 & \\
\hline CT-abs. 0-0 peak width [meV] & 147 & \\
\hline Peaks \# [-] & 3 & \\
\hline
\end{tabular}

Table S2. Parameters used in the vibronic progressions of the model fits to the CT-EL and absorption spectra in Figure 3 of the main text. The CT-EL 0-0 peak width was obtained by fitting a Gaussian peak to the simulated 0-0 spectrum that was calculated by kMC using the parameters in Table S1, c.f. Figure 4 in main text. The CTabsorption 0-0 peak width follows by convolution of the LUMO and HOMO disorder values as explained in the main text. Phonon energies and the number of peaks follow Ref. ${ }^{21}$ The Huang-Rhys factor $S$ was manually fitted.

\begin{tabular}{|c|c|c|}
\hline & & \\
\hline & TQ1:PC ${ }_{71} B M$ & PCDTBT:PC ${ }_{61} B M$ \\
\hline$E_{0}[\mathrm{eV}]$ & 1.81 & 1.88 \\
\hline$k T_{0}[\mathrm{meV}]$ & 35 & 45 \\
\hline
\end{tabular}

Table S3. Fitting parameters of equation 6 in the main text (Urbach tail to phenomenologically account for the donor $\mathbf{S 1}$ absorption) used in Figure 3 of the main text. 
(1) Johs, B.; Hale, J. S. Dielectric Function Representation by B-Splines. Phys Stat Sol A 2008, 205 (4), 715-719. https://doi.org/10.1002/pssa.200777754.

(2) Burkhard, G. F.; Hoke, E. T.; McGehee, M. D. Accounting for Interference, Scattering, and Electrode Absorption to Make Accurate Internal Quantum Efficiency Measurements in Organic and Other Thin Solar Cells. Adv. Mater. 2010, 22 (30), 3293-3297. https://doi.org/10.1002/adma.201000883.

(3) Vandewal, K.; Goris, L.; Haenen, K.; Geerts, Y.; Manca, J. V. Highly Sensitive Spectroscopic Characterization of Inorganic and Organic Heterojunctions for Solar Cells. Eur. Phys. J. Appl. Phys. 2006, 36 (3), 281-283. https://doi.org/10.1051/epjap:2006140.

(4) Vandewal, K.; Albrecht, S.; Hoke, E. T.; Graham, K. R.; Widmer, J.; Douglas, J. D.; Schubert, M.; Mateker, W. R.; Bloking, J. T.; Burkhard, G. F. et al., A. Efficient Charge Generation by Relaxed Charge-Transfer States at Organic Interfaces. Nat. Mater. 2014, 13 (1), 63-68. https://doi.org/10.1038/nmat3807.

(5) Buchaca-Domingo, E.; Vandewal, K.; Fei, Z.; Watkins, S. E.; Scholes, F. H.; Bannock, J. H.; de Mello, J. C.; Richter, L. J.; DeLongchamp, D. M.; Amassian et al., Direct Correlation of Charge Transfer Absorption with Molecular Donor:Acceptor Interfacial Area via Photothermal Deflection Spectroscopy. J. Am. Chem. Soc. 2015, 137 (16), 5256-5259. https://doi.org/10.1021/ja512410f.

(6) Melianas, A.; Pranculis, V.; Devižis, A.; Gulbinas, V.; Inganäs, O.; Kemerink, M. DispersionDominated Photocurrent in Polymer:Fullerene Solar Cells. Adv. Funct. Mater. 2014, 24 (28), 4507-4514. https://doi.org/10.1002/adfm.201400404.

(7) Abramavicius, V.; Pranculis, V.; Melianas, A.; Inganäs, O.; Gulbinas, V.; Abramavicius, D. Role of Coherence and Delocalization in Photo-Induced Electron Transfer at Organic Interfaces. Sci. Rep. 2016, 6 (1), 32914. https://doi.org/10.1038/srep32914.

(8) Lane, P. A.; Cunningham, P. D.; Melinger, J. S.; Esenturk, O.; Heilweil, E. J. Hot Photocarrier Dynamics in Organic Solar Cells. Nat. Commun. 2015, 6 (1), 7558. https://doi.org/10.1038/ncomms8558.

(9) Melianas, A.; Etzold, F.; Savenije, T. J.; Laquai, F.; Inganäs, O.; Kemerink, M. Photo-Generated Carriers Lose Energy during Extraction from Polymer-Fullerene Solar Cells. Nat. Commun. 2015, 6, 8778. https://doi.org/10.1038/ncomms9778.

(10) Tang, Z.; George, Z.; Ma, Z.; Bergqvist, J.; Tvingstedt, K.; Vandewal, K.; Wang, E.; Andersson, L. M.; Andersson, M. R.; Zhang, F. et al., O. Semi-Transparent Tandem Organic Solar Cells with 90\% Internal Quantum Efficiency. Adv. Energy Mater. 2012, 2 (12), 1467-1476. https://doi.org/10.1002/aenm.201200204.

(11) Kurpiers, J.; Ferron, T.; Roland, S.; Jakoby, M.; Thiede, T.; Jaiser, F.; Albrecht, S.; Janietz, S.; Collins, B. A.; Howard, I. A. et al., Probing the Pathways of Free Charge Generation in Organic Bulk Heterojunction Solar Cells. Nat. Commun. 2018, 9 (1), 2038. https://doi.org/10.1038/s41467-018-04386-3.

(12) Barker, A. J.; Chen, K.; Hodgkiss, J. M. Distance Distributions of Photogenerated Charge Pairs in Organic Photovoltaic Cells. J. Am. Chem. Soc. 2014, 136 (34), 12018-12026. https://doi.org/10.1021/ja505380j.

(13) Dimitrov, S. D.; Azzouzi, M.; Wu, J.; Yao, J.; Dong, Y.; Tuladhar, P. S.; Schroeder, B. C.; Bittner, E. R.; McCulloch, I.; Nelson, J. et al., Spectroscopic Investigation of the Effect of Microstructure and Energetic Offset on the Nature of Interfacial Charge Transfer States in Polymer: Fullerene Blends. J. Am. Chem. Soc. 2019, 141 (11), 4634-4643. https://doi.org/10.1021/jacs.8b11484.

(14) Gélinas, S.; Rao, A.; Kumar, A.; Smith, S. L.; Chin, A. W.; Clark, J.; van der Poll, T. S.; Bazan, G. C.; Friend, R. H. Ultrafast Long-Range Charge Separation in Organic Semiconductor Photovoltaic Diodes. Science 2014, 343 (6170), 512-6. https://doi.org/10.1126/science.1246249. 
(15) Ye, L.; Hu, H.; Ghasemi, M.; Wang, T.; Collins, B. A.; Kim, J.-H.; Jiang, K.; Carpenter, J. H.; Li, H.; $\mathrm{Li}$, Z. et al., Quantitative Relations between Interaction Parameter, Miscibility and Function in Organic Solar Cells. Nat. Mater. 2018, 17 (3), 253-260. https://doi.org/10.1038/s41563-0170005-1.

(16) Bergqvist, J.; Lindqvist, C.; Bäcke, O.; Ma, Z.; Tang, Z.; Tress, W.; Gustafsson, S.; Wang, E.; Olsson, E.; Andersson, M. R. et al., C. Sub-Glass Transition Annealing Enhances Polymer Solar Cell Performance. J. Mater. Chem. A 2014, 2 (17), 6146-6152. https://doi.org/10.1039/C3TA14165A.

(17) Vandewal, K.; Tvingstedt, K.; Gadisa, A.; Inganäs, O.; Manca, J. V. Relating the Open-Circuit Voltage to Interface Molecular Properties of Donor:Acceptor Bulk Heterojunction Solar Cells. Phys. Rev. B 2010, 81 (12), 125204. https://doi.org/10.1103/PhysRevB.81.125204.

(18) Wilken, S.; Upreti, T.; Melianas, A.; Dahlström, S.; Persson, G.; Olsson, E.; Österbacka, R.; Kemerink, M. Experimentally Calibrated Kinetic Monte Carlo Model Reproduces Organic Solar Cell Current-Voltage Curve. unpublished.

(19) Etzold, F.; Howard, I. A.; Mauer, R.; Meister, M.; Kim, T.-D.; Lee, K.-S.; Baek, N. S.; Laquai, F. Ultrafast Exciton Dissociation Followed by Nongeminate Charge Recombination in PCDTBT:PCBM Photovoltaic Blends. J. Am. Chem. Soc. 2011, 133 (24), 9469-9479. https://doi.org/10.1021/ja201837e.

(20) Felekidis, N.; Melianas, A.; Kemerink, M. Automated Open-Source Software for Charge Transport Analysis in Single-Carrier Organic Semiconductor Diodes. Org. Electron. 2018, 61, 318-328. https://doi.org/10.1016/j.orgel.2018.06.010.

(21) Melianas, A.; Felekidis, N.; Puttisong, Y.; Meskers, S. C. J.; Inganäs, O.; Chen, W. M.; Kemerink, M. Nonequilibrium Site Distribution Governs Charge-Transfer Electroluminescence at Disordered Organic Heterointerfaces. Proc. Natl. Acad. Sci. 2019, 116 (47), 23416-23425. https://doi.org/10.1073/pnas.1908776116. 